\title{
Radical Intermediates in the Degradation of Hop Acids
}

Jan K. E. T. Berton ${ }^{\mathrm{a}}$, Yannis Verbeke ${ }^{\mathrm{a}, \mathrm{b}}$, Bo Van Durme ${ }^{\mathrm{a}, \mathrm{c}}$, Kevin Huvaere $^{\mathrm{a}, \mathrm{d}^{*}}$

a EcoSynth NV, Industrielaan 12, 9800 Deinze, Belgium

${ }^{\mathrm{b}}$ Trainee affiliated to the Odisee University of Applied Sciences, Department of Chemistry, Ghent, Belgium (associated to the Catholic University of Leuven)

'Trainee affiliated to Ghent University, Faculty of Sciences, Department of Chemistry, Ghent, Belgium

d Plinius Labs NV, Venecolaan 50G, 9880 Aalter, Belgium

*khuvaere@ecosynth.be; kevin.huvaere@pliniuslabs.com 


\section{Contents}

Radical formation during ageing ( $12 \mathrm{~d}$ ) of alkaline solutions of isohumulones and derivatives............ 3

Radical formation in alkaline solutions of isohumulones and derivatives .......................................... 4

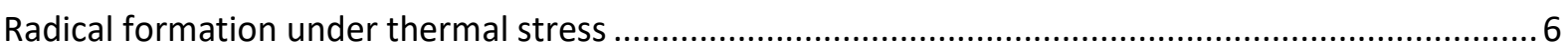

Radical formation in the presence of transition metals ........................................................... 7

Radical formation in the presence of hydrogen peroxide ................................................................. 
Radical formation during ageing (12 d) of alkaline solutions of isohumulones and derivatives

Ageing alkaline solution of isohumulones (or its reduced derivatives), for 12 days at room temperature or at $40^{\circ} \mathrm{C}$, under ambient atmosphere, gave detectable signals in EPR spectroscopy after samples were treated with DMPO spin trap with subsequent incubation $\left(2 \mathrm{~h}\right.$ at $60^{\circ} \mathrm{C}$, under nitrogen atmosphere)

Table S1. Spin parameters of hop acids (as potassium salts) kept for 12 days under air at room temperature or at $40^{\circ} \mathrm{C}$. Samples were measured after $2 \mathrm{~h}$ of incubation under nitrogen atmosphere using DMPO as spin trap.

\begin{tabular}{|c|c|c|c|c|c|c|c|c|c|}
\hline \multirow[b]{3}{*}{ Substrate $^{a}$} & \multicolumn{8}{|c|}{ Simulation parameters } & \multirow[b]{3}{*}{ ID c } \\
\hline & \multicolumn{4}{|c|}{ Room temperature } & \multicolumn{4}{|c|}{$40^{\circ} \mathrm{C}$} & \\
\hline & $a_{N}(G)$ & $a_{H}(G)$ & $a_{H^{v}}(G)$ & Conc. $(\mu \mathrm{M})^{b}$ & $a_{N}(G)$ & $a_{H}(G)$ & $a_{H}{ }^{v}(G)$ & Conc. $(\mu \mathrm{M})$ & \\
\hline \multirow[t]{4}{*}{ Iso } & 15.1 & 22.3 & - & 3.3 & 15.2 & 22.4 & - & 6.4 & $\cdot R$ \\
\hline & 14.3 & - & - & 2.1 & 14.3 & - & - & 1.3 & $x$ \\
\hline & 13.7 & 10.8 & 1.2 & 2.8 & 14.6 & 11.6 & - & 3.6 & $\cdot \mathrm{OOR}$ \\
\hline & 12.0 & 8.8 & 1.8 & 0.5 & 12.0 & 8.5 & 2.1 & 0.2 & $\cdot \mathrm{OR}$ \\
\hline \multirow[t]{4}{*}{ Dihydro } & 15.1 & 22.1 & - & 4.8 & 15.2 & 22.9 & - & 8.1 & $\cdot R$ \\
\hline & 15.3 & 23.6 & - & 2.8 & & & & & $\cdot R$ \\
\hline & 14.3 & - & - & 4.5 & 14.3 & - & - & 4.5 & $x$ \\
\hline & 12.2 & 8.1 & 2.5 & 0.5 & 12.1 & 8.4 & 2.3 & 0.7 & •OR \\
\hline \multirow[t]{4}{*}{ Tetra } & 15.3 & 23.1 & - & 5.6 & 15.3 & 23.2 & - & 2.9 & $\cdot R$ \\
\hline & & & & & 14.9 & 22.3 & - & 3.9 & $\bullet R$ \\
\hline & 14.5 & - & - & 2.9 & 14.5 & - & - & 3.2 & $x$ \\
\hline & 14.8 & 18.1 & - & 1.1 & 14.8 & 18.4 & - & 0.8 & $\cdot C(O) R$ \\
\hline \multirow[t]{4}{*}{ Humulones } & 15.0 & 22.7 & - & 3.5 & 15.0 & 22.5 & - & 4.7 & $\cdot R$ \\
\hline & 14.2 & - & - & 1.9 & 14.3 & - & - & 1.8 & $x$ \\
\hline & 16.0 & 23.0 & - & 0.4 & 16.3 & 22.8 & - & 0.2 & $\cdot R$ \\
\hline & 14.5 & 10.9 & 2.6 & 6.2 & 14.0 & 11.6 & 1.8 & 4.0 & •OOR \\
\hline \multirow[t]{5}{*}{ Lupulones } & 15.2 & 23.1 & - & 2.7 & 15.2 & 22.6 & - & 7.7 & $\cdot R$ \\
\hline & 14.3 & - & - & 2.1 & 14.4 & - & - & 1.8 & $x$ \\
\hline & 15.5 & 22.1 & - & 5.1 & 13.8 & 20.6 & - & 1.4 & $\cdot R$ \\
\hline & 14.2 & 10.0 & 2.3 & 1.4 & 14.6 & 10.7 & 3.1 & 4.0 & •OOR \\
\hline & 14.6 & 18.1 & - & 0.6 & 14.7 & 18.0 & - & 0.4 & ${ }^{\circ} \mathrm{C}(\mathrm{O}) \mathrm{R}$ \\
\hline
\end{tabular}

${ }^{a}$ Iso, Dihydro, and Tetra refers to isohumulones, dihydroisohumulones, and tetrahydroisohumulones, respectively. All substrates prevail as potassium salts. ${ }^{b}$ Concentration. ${ }^{c}$ Identification of trapped radical: $\cdot R=$ carbon-centred alkyl, ${ }^{\circ} \mathrm{C}(O) R=$ acyl, ${ }^{\circ} \mathrm{O} R=$ alkoxyl, ${ }^{\circ} \mathrm{OO} R=$ peroxyl, $X$ = spin adduct decay. 


\section{Radical formation in alkaline solutions of isohumulones and derivatives}

Addition of DMPO to an alkaline solution of isohumulones (or its reduced derivatives), continuously monitored by EPR spectroscopy for an extended period $(\sim 10 \mathrm{~h})$, showed that spin adduct formation readily occurred. All derivatives of isohumulones produced detectable species after a couple of hours, most of which were attributed to the trapping of carbon-centred radicals (Table S1). The radicals must originate in the hop acids since an alkaline solution of DMPO, but in the absence of substrate, did not produce detectable signals.

Table S2. Detailed spin parameters, as extracted from simulating spectra of the DMPO adducts formed under ambient conditions (room temperature, air) in alkaline solutions of isohumulones and its derivatives, measured $16 \mathrm{~h}$ after addition of spin trap.

${ }^{a}$ Iso, Dihydro, and Tetra refers to the potassium salts of isohumulones, dihydroisohumulones, and tetrahydroisohumulones, respectively. ${ }^{b}$ Linewidth. ${ }^{c}$ Concentration. ${ }^{d}$ Identification of trapped radical: $R^{*}=$ carbon-centred alkyl, $X=$ spin adduct decay.

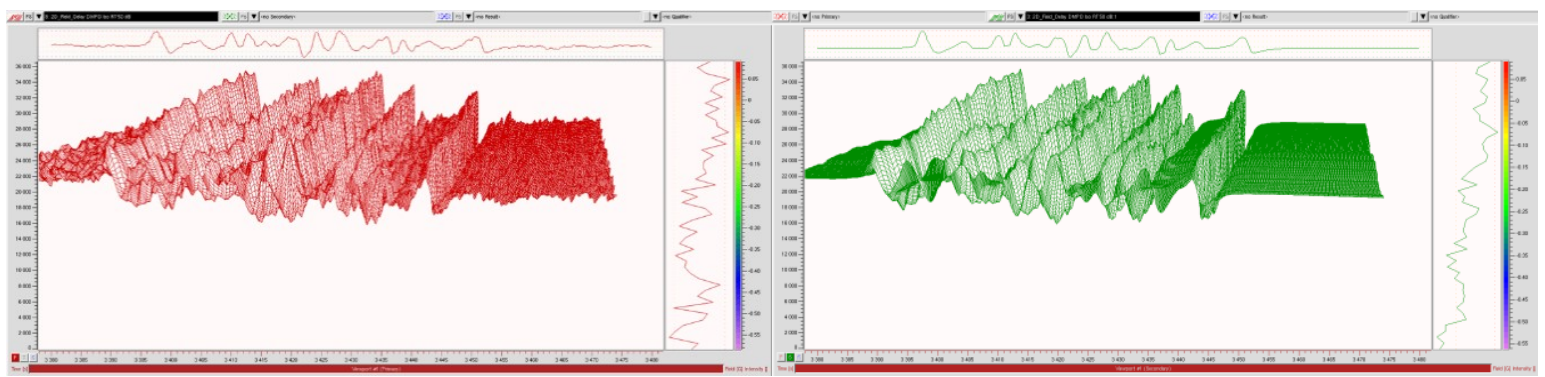

Figure S1. 2D EPR experimental spectrum (left panel) and simulation thereof (right panel) of an alkaline formulation of isohumulones monitored up to 10 hours after addition of DMPO as spin trap and start of EPR measurement.

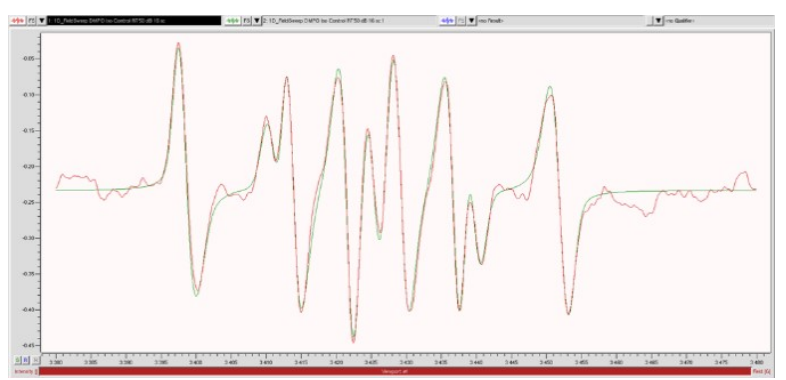

Figure S2. 1D EPR experimental spectrum (red trace) and simulation thereof (green trace) of an alkaline formulation of isohumulones taken 16 hours after addition of DMPO as spin trap. 


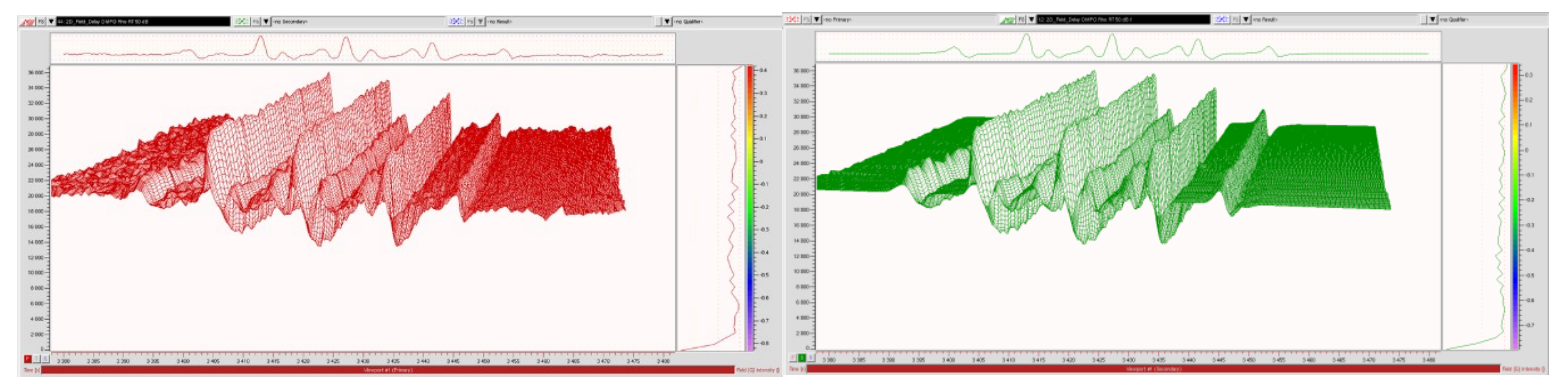

Figure S3. 2D EPR experimental spectrum (left panel) and simulation thereof (right panel) of an alkaline formulation of dihydroisohumulones monitored up to 10 hours after addition of DMPO as spin trap and start of EPR measurement.

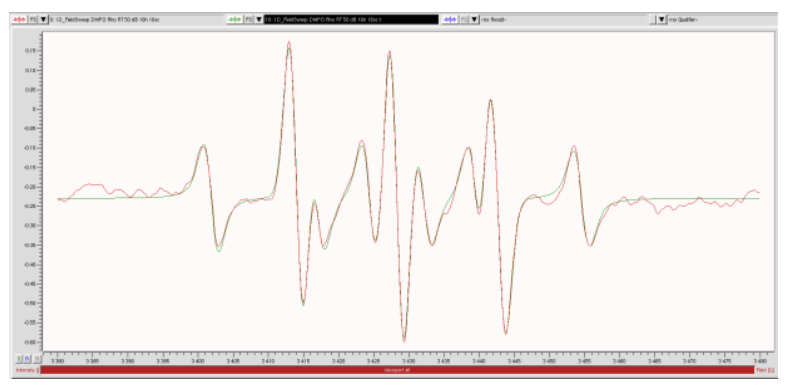

Figure S4. 1D EPR experimental spectrum (red trace) and simulation thereof (green trace) of an alkaline formulation of dihydroisohumulones taken 16 hours after addition of DMPO as spin trap.

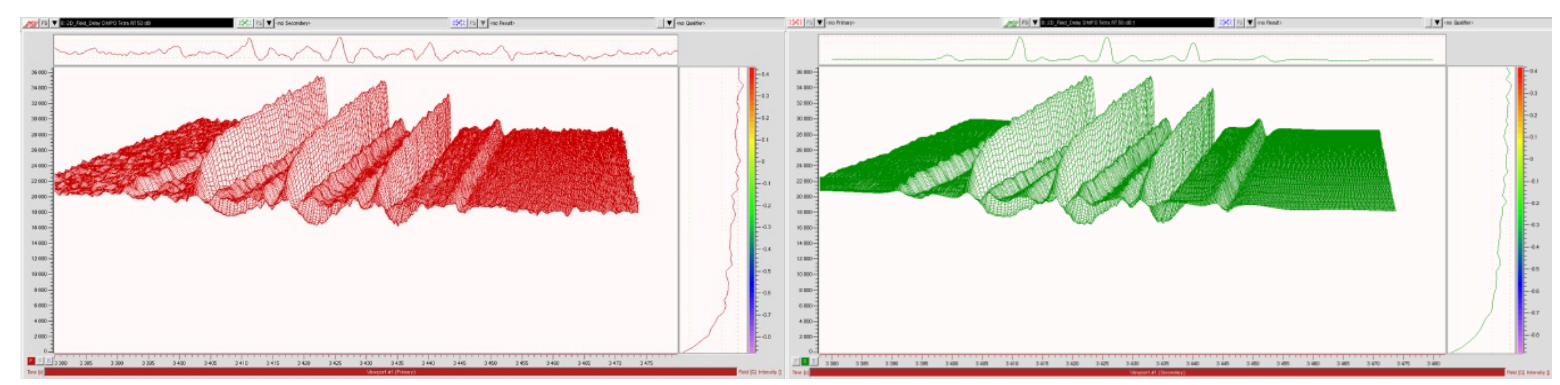

Figure S5. 2D EPR experimental spectrum (left panel) and simulation thereof (right panel) of an alkaline formulation of tetrahydroisohumulones monitored up to 10 hours after addition of DMPO as spin trap and start of EPR measurement.

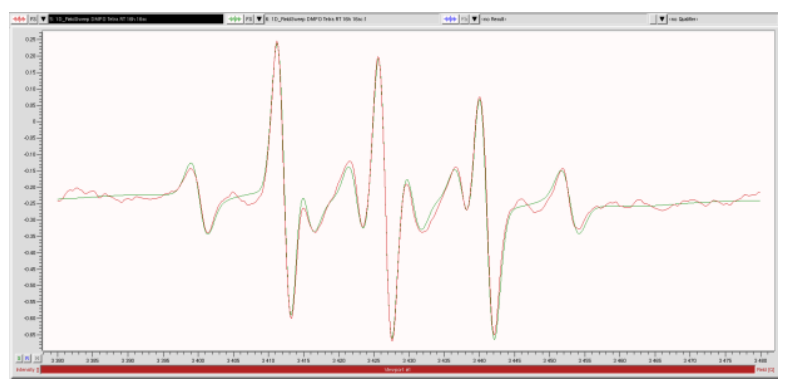

Figure S6. 1D EPR experimental spectrum (red trace) and simulation thereof (green trace) of an alkaline formulation of tetrahydroisohumulones taken 16 hours after addition of DMPO as spin trap. 


\section{$\underline{\text { Radical formation under thermal stress }}$}

When sample was not aged, but spin trap was added and the mixture was kept at $60^{\circ} \mathrm{C}$ signals were readily observed. Under nitrogen, generally a more intense signal is obtained. Mainly isohumulones and humulinones gave intense signals with PBN, while dihydroisohumulones gave spectra of lower intensity. With DMPO as spin trap, no relevant adducts were detected.

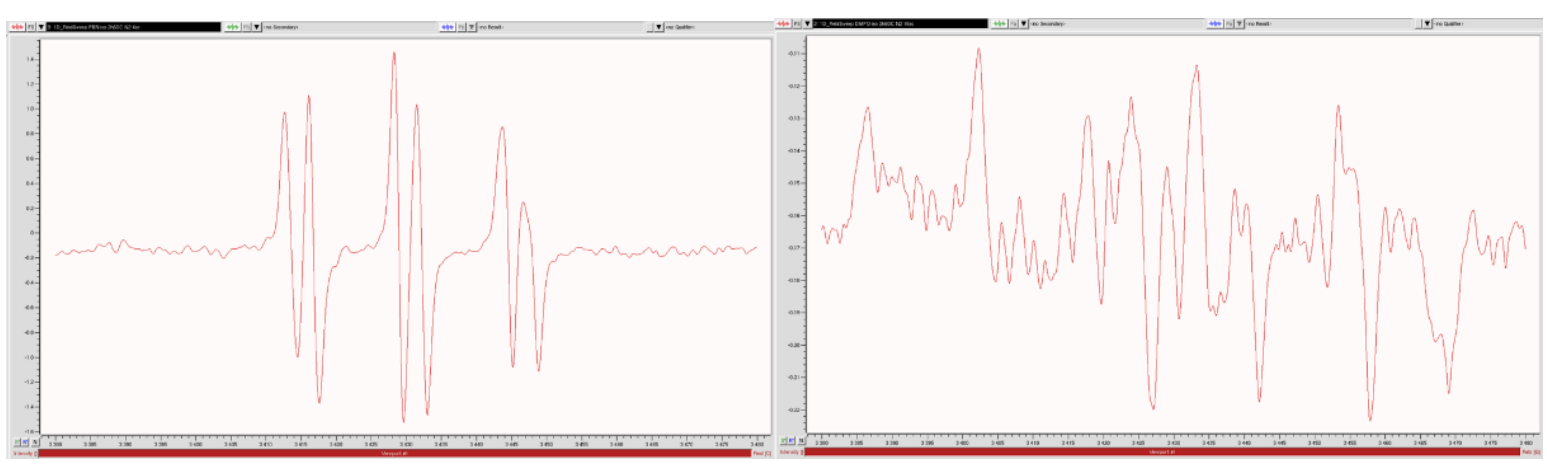

Figure S7. 1D EPR experimental spectrum of isohumulones in the presence of PBN (left trace) and DMPO (right trace), after 2 hours of thermal stress $\left(60^{\circ} \mathrm{C}\right)$ under nitrogen atmosphere.

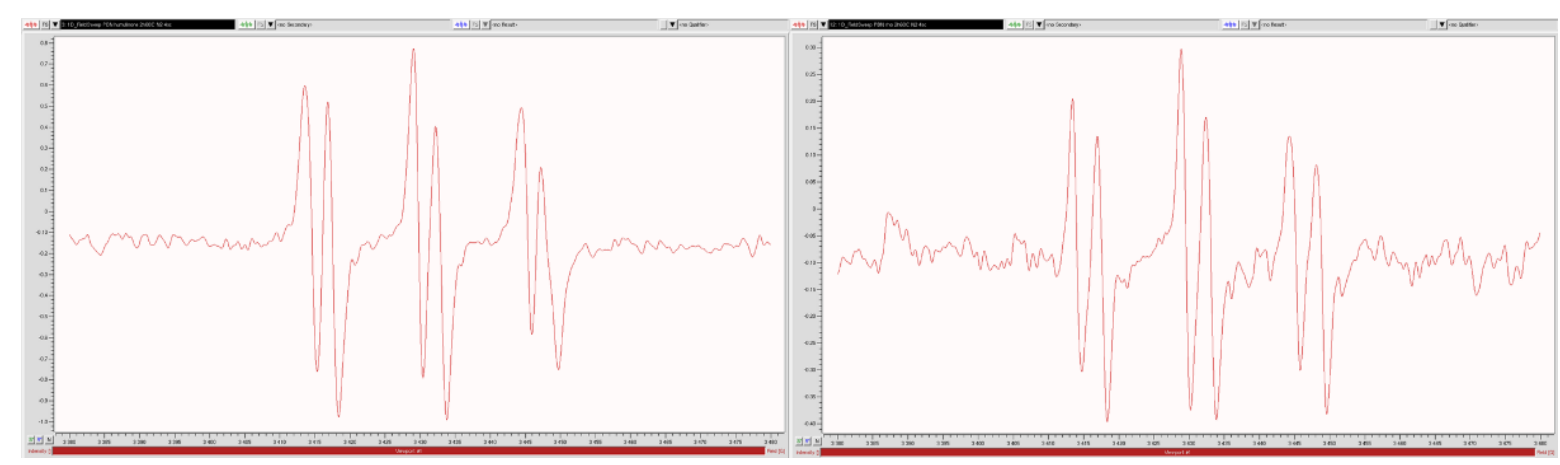

Figure S8. 1D EPR experimental spectrum of humulinones (left trace) and dihydroisohumulones (right trace) in the presence of PBN, after 2 hours of thermal stress $\left(60^{\circ} \mathrm{C}\right)$ under nitrogen atmosphere. 


\section{$\underline{\text { Radical formation in the presence of transition metals }}$}

An intense EPR signal was observed when ferrous iron (Fe") was added to a DMPO solution. Simulation, showed high concentrations $(27 \mu \mathrm{M})$ of a trapped carbon radical with $\mathrm{a}_{\mathrm{N}} \sim 15.1 \mathrm{G}, \mathrm{a}_{\mathrm{H}} \sim 22.1 \mathrm{G}(\mathrm{LW} 1.8$ $\mathrm{G}, \mathrm{g} \sim 2$ 2.0053). The corresponding experiments with PBN as trap could not produce detectable adducts, not even after prolonged contact $(\sim 20 \mathrm{~h})$. In the presence of hop acids, PBN adducts were readily formed for isohumulones, whereas for dihydroisohumulones signals were only observed after prolonged reaction time.

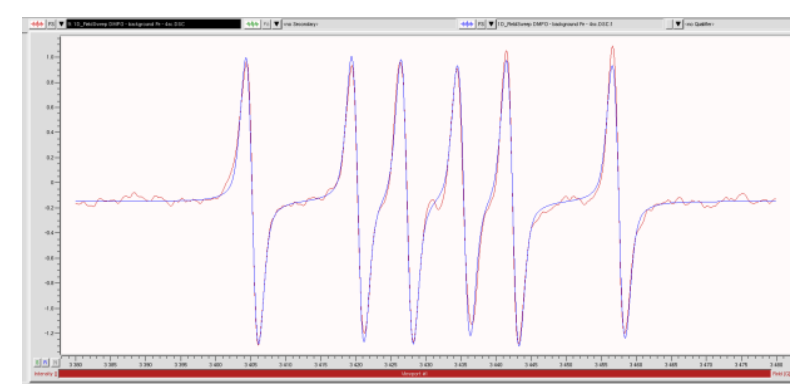

Figure S9. 1D EPR experimental spectrum (red trace) and simulation thereof (blue trace) of an aqueous ferrous ion solution (approximately $0.35 \mathrm{mM}$ ) in the presence of DMPO as spin trap. Spectrum was taken immediately after mixing.

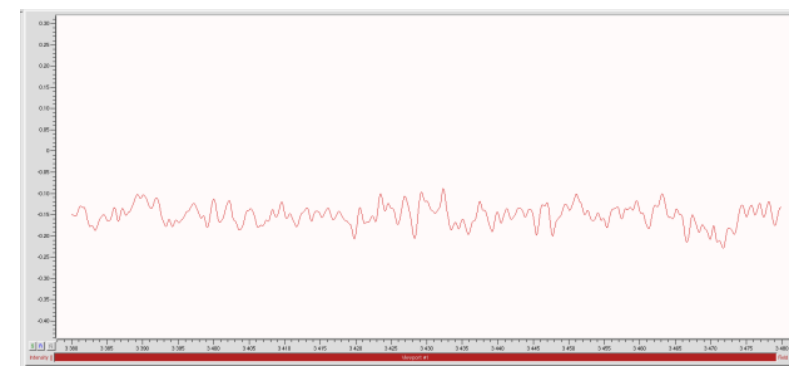

Figure S10. 1D EPR experimental spectrum of an aqueous ferrous ion solution (approximately $0.35 \mathrm{mM}$ ) in the presence of PBN as spin trap. Spectrum was taken approximately $20 \mathrm{~h}$ after mixing.

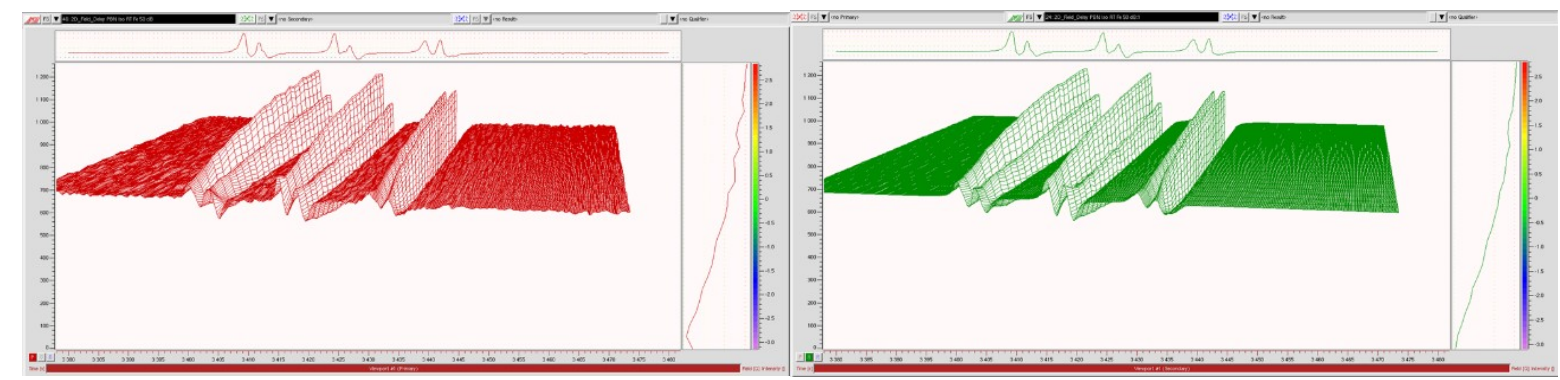

Figure S11. 2D EPR experimental spectrum (left panel) and simulation thereof (right panel) of an alkaline formulation of isohumulones in the presence of PBN as spin trap, monitored for approximately $25 \mathrm{~min}$ after addition of ferrous ions. 


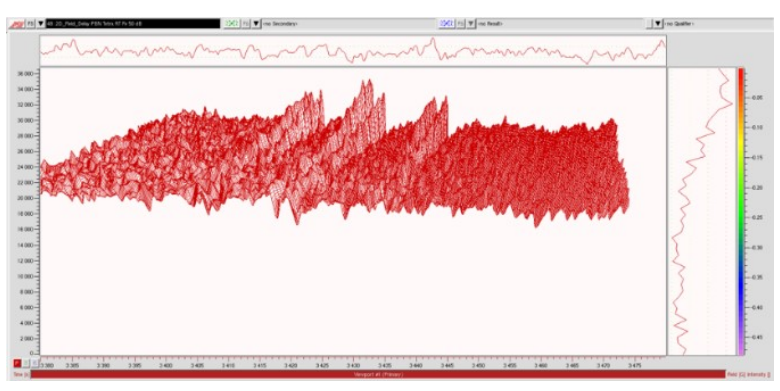

Figure S12. 2D EPR experimental spectrum of an alkaline formulation of dihydroisohumulones in the presence of PBN as spin trap, monitored for approximately 10 hours after addition of ferrous ions. 


\section{Radical formation in the presence of hydrogen peroxide}

Hydrogen peroxide in the presence of DMPO gave a spectrum with adducts $\mathrm{a}_{\mathrm{N}} \sim 13.7 \mathrm{G}, \mathrm{a}_{\mathrm{H}} \sim 11.5 \mathrm{G}$, and $\mathrm{a}_{\mathrm{H}}{ }^{\nu} \sim 1.4 \mathrm{G}$, assigned to hydroperoxyl radical, ${ }^{\circ} \mathrm{OOH}$, trapping and a hydroxyl radical, ${ }^{\circ} \mathrm{OH}$ with $\mathrm{a}_{\mathrm{N}}$ $\left.\sim 13.4 \mathrm{G}, \mathrm{a}_{\mathrm{H}} \sim 15.1 \mathrm{G}\right)$. PBN did not give any adducts with only hydrogen peroxide.

When hop acids were present, other radicals readily formed for isohumulones (and also dihydroisohumulones), but not for tetrahydroisohumulones which failed to produce adducts.



Figure S13. 1D EPR experimental spectrum of an aqueous hydrogen peroxide solution in the presence of DMPO as spin trap. Spectrum was taken immediately after mixing. Solution details are: $25 \mu \mathrm{l} 0.5 \mathrm{M}$ DMPO (in MeCN), $25 \mu \mathrm{l} \mathrm{H}_{2} \mathrm{O}$, and $50 \mu \mathrm{l} 35 \%$ $\mathrm{H}_{2} \mathrm{O}_{2}$.
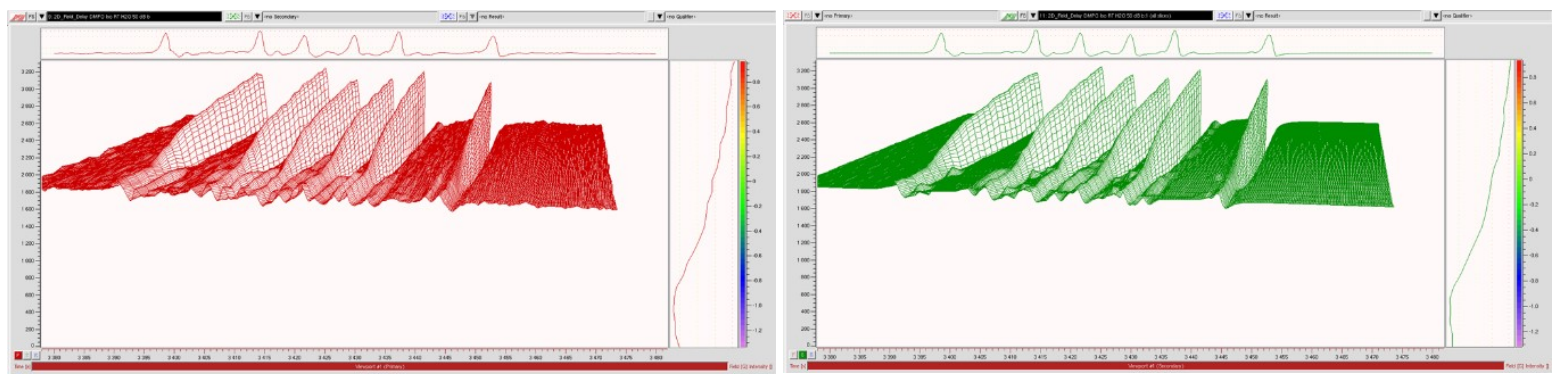

Figure S14. 2D EPR experimental spectrum (left panel) and simulation thereof (right panel) of an alkaline formulation of isohumulones in the presence of DMPO as spin trap, monitored for approximately 60 min after addition of hydrogen peroxide.
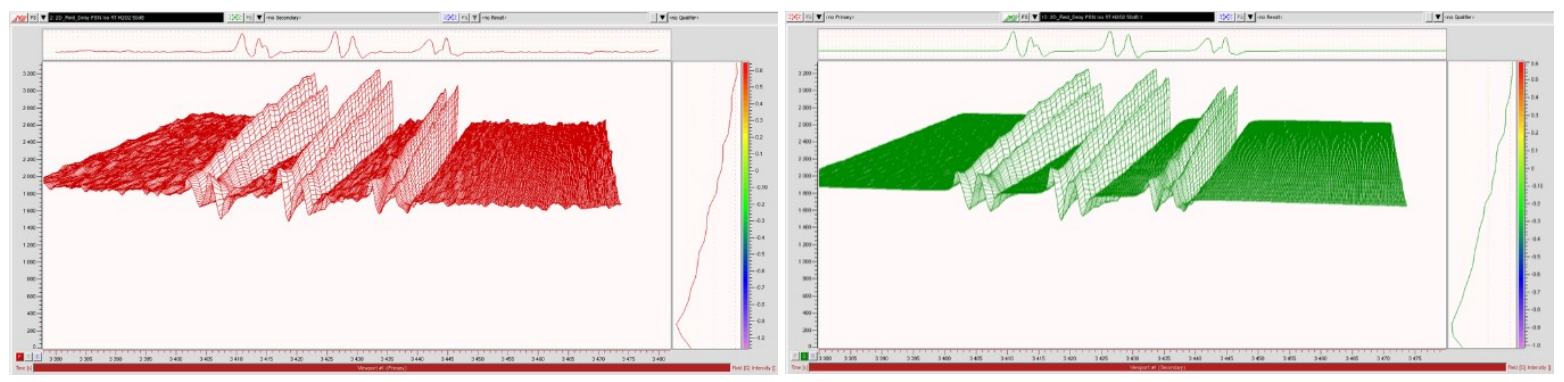

Figure S15. 2D EPR experimental spectrum (left panel) and simulation thereof (right panel) of an alkaline formulation of isohumulones in the presence of PBN as spin trap, monitored for approximately $60 \mathrm{~min}$ after addition of hydrogen peroxide. 


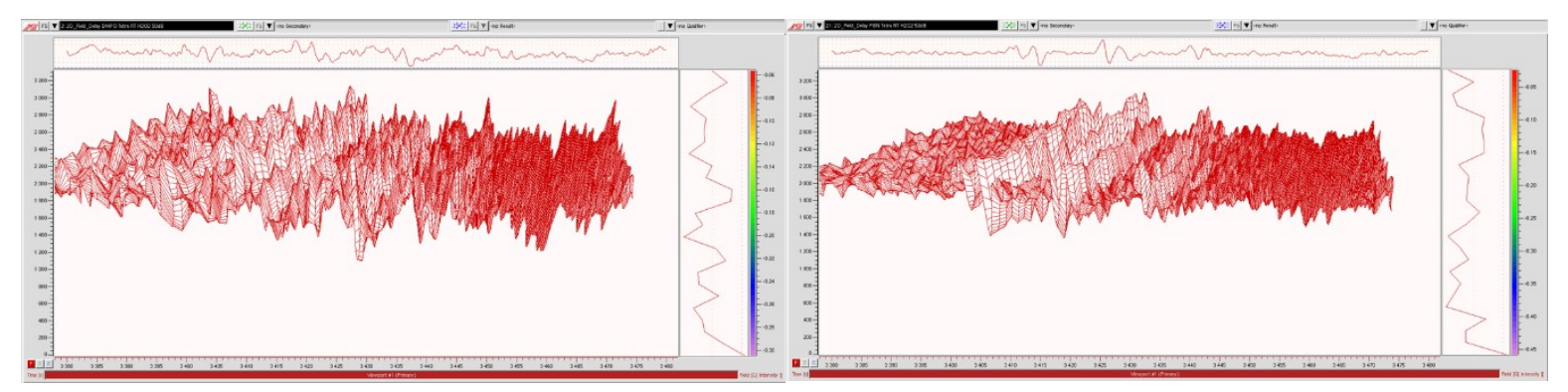

Figure S16. 2D EPR experimental spectrum of an alkaline formulation of tetrahydroisohumulones in the presence of DMPO (left panel) and PBN (right panel) as spin trap, monitored for approximately $60 \mathrm{~min}$ after addition of hydrogen peroxide. 